\title{
The New Small Wheel Detector Control System of the ATLAS experiment
}

\author{
Christos Paraskevopoulos ${ }^{a, b, *}$ on behalf of the ATLAS Muon Group \\ ${ }^{a}$ National Technical University of Athens, \\ Heroon Polytechniou 9, Zografou, Greece \\ ${ }^{b}$ Brookhaven National Laboratory, \\ Upton, NY 11973-5000, U.S.A. \\ E-mail: christos.paraskevopoulos@cern.ch
}

\begin{abstract}
A series of upgrades are planned for the LHC accelerator to increase its instantaneous luminosity to $7.5 \times 10^{34} \mathrm{~cm}^{-2} \mathrm{~s}^{-1}$. The luminosity increase drastically impacts the ATLAS trigger and readout data rates. The present ATLAS Small Wheel Muon detector will be replaced with the New Small Wheel detector which is expected to be installed in the ATLAS underground cavern by the end of the Long Shutdown 2 of the LHC. Due to its complexity and long-term operation, the New Small Wheel requires the development of a sophisticated Detector Control System. The use of such a system is necessary to allow the detector to function consistently and safely as well as to function as a seamless interface to all sub-detectors and the technical infrastructure of the experiment. The central system handles the transition between the probes possible operating states while ensuring continuous monitoring and archiving of the systems operating parameters. Any abnormality in any subsystem of the detector triggers a signal or alarm, which alerts the user and either adapts to automatic processes or allows manual actions to reset the system to function properly.
\end{abstract}

The Ninth Annual Conference on Large Hadron Collider Physics - LHCP2021

7-12 June 2021

Online

${ }^{*}$ Speaker 


\section{ATLAS New Small Wheel}

ATLAS [1] is the largest high-energy physics detector ever built by man. The LHC delivers millions of collisions each second, that take place in the heart of ATLAS. These collisions though, create a dangerous radiation environment, which the detector has to endure. To efficiently handle the increased luminosity of the High-Luminosity LHC (HL-LHC), the Small Wheel (SW) which is the first station of the ATLAS muon spectrometer end-cap system, will be replaced. The New Small Wheel (NSW) [2] will have to operate in a high background radiation region (up to $22 \mathrm{kHz} / \mathrm{cm}^{2}$ ) while reconstructing muon tracks with high precision as well as providing information for the Level-1 trigger. The New Small Wheel consists of two detector technologies of gaseous detectors, the first is called small-strip Thin Gap Chambers (sTGCs), and the second comes from the category of micro-pattern gaseous detectors and is named Micromegas (MMG) [3]. The NSW apart from the two new detector technologies is a platform presenting new Custom ASICs and electronic boards, new readout system based on Front End LInk eXchange (FELIX), 2.5 Million readout channels, common configuration, calibration \& Detector Control System (DCS) path and new power supply system.

\section{The New Small Wheel Detector Control System}

In order to monitor and control the operation of the detector, a framework has been devised, which allows for remote supervision and intervention in the detector and its sub-systems: The Detector Control System (DCS) [4].The DCS is simply a Supervisory Control And Data Acquisition (SCADA) system equipped with User Interfaces (UIs), automated scripts and control/monitor functionality. This control scheme is used daily, in the ATLAS Control Room. It is also used by the subdetector experts to guarantee a safe and efficient Physics run. The main task of the DCS is to enable the coherent and safe operation of the full detector by continuously monitoring its operational parameters and its overall state.

\subsection{System Architecture}

The NSW DCS projects closely follow the existing look, feel and command structure of MUON DCS, to facilitate the shifter and expert operations. The projects are mapped onto a hierarchy of Finite State Machine (FSM) elements using the Joint COntrols Project (JCOP) FSM toolkit [5].
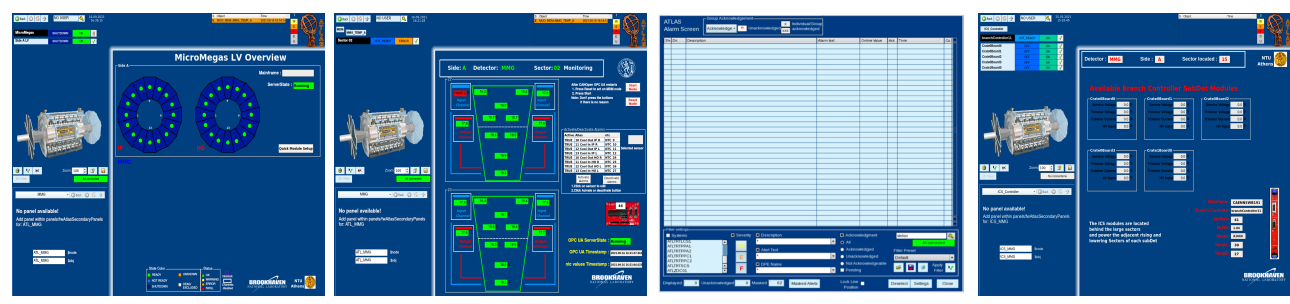

Figure 1: Various monitoring panels, MMG Low Voltage Overview, Temperature monitoring, the alarm screen and other controls of NSW 
The FSM is conceived as an abstract machine that is able to be in only one of a finite number of states at a time. The state can change when initiated by a triggering event or condition (transition state).

The top node of both MMG and sTGC will propagate its state and receive commands from the ATLAS overall DCS (Fig.2). Shifters will mainly use the DCS FSM Overview and DCS Alarm Screen (Fig.1).

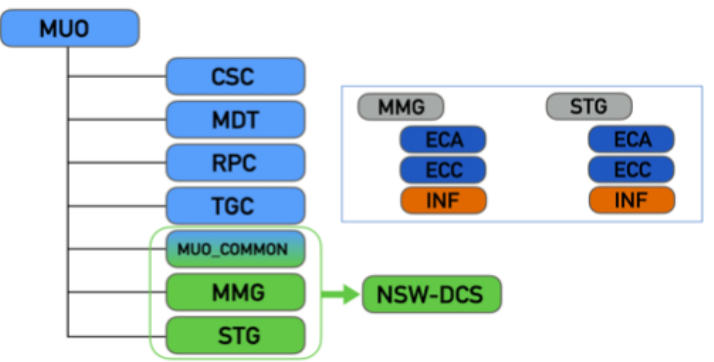

Figure 2: The overview of the ATLAS MUO DCS structure with NSW DCS integration and brief structure of the sub-detector node structure.

\subsection{Projects}

\subsubsection{Power Supply}

High and Low voltages for MMG and sTGC are supplied by the widely used CAEN system [6], which is developed for the LHC experiments. Among its core features are:

- Remote control

- Split of radiation sensitive parts from parts that can be housed in a hostile area

- Compatibility with operation under radiation and magnetic field as in the ATLAS cavern

Communication with the control machines is achieved through OPC UA server-client connection. Side A and C, NSW OPC UA servers, are deployed on the host servers while the OPC UA clients, running on the same machines, gather the address space of the individual channel parameters and transmit them to the projects. The system architecture consists of mainframes, which house the branch controllers, connected with the Low Voltage (LV) and High Voltage (HV) crates. Intermediate Conversion Stage (ICS) modules provide the Low Voltage for the readout cards. ICS will be in the "hostile" area of the system near to the chambers, mounted on the NSW. Eleven CAEN mainframes of type SY4527 will control all LV and HV for NSW monitored and controlled by FSM panels (Fig.3).

Each HV branch controller is connected with a set of Embedded Assembly SYstem (EASY) crates, which are connected as a daisy-chain. HV Crates and boards are supplied with an external DC power of $48 \mathrm{~V}$ generated by separate AC-DC converters, while the LV ICS crates are powered from primary generators with adjustable voltage output. The mainframes, generators and branch controllers are located in ATLAS underground service areas while EASY crates and boards are in the ATLAS cavern. 

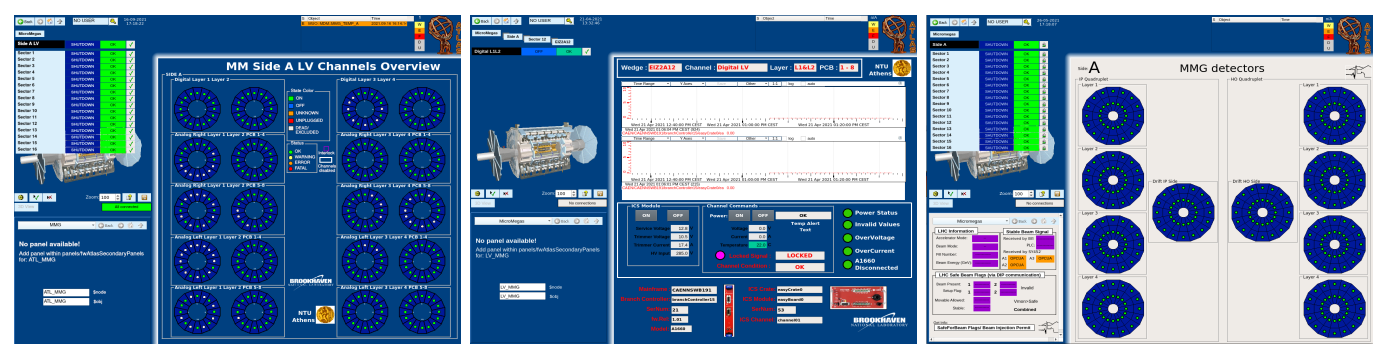

Figure 3: Power supply Control and monitoring FSM panels

\subsubsection{Electronics Monitoring}

The NSW is a fully redundant trigger and tracking detector system, adequately supported by an advanced electronics scheme and ready to handle the challenges of increased instantaneous luminosity at the High Luminosity LHC. Its separate configuration/monitor, readout and trigger path consists of 2.4 million readout channels that result in 100000 DCS parameters. The NSW electronics for the Trigger and Data Acquisition (TDAQ) path of both detectors is divided into two major categories, on-detector and off-detector electronics and the DCS, configuration and calibration back-ends monitored through FSM (Fig.4).
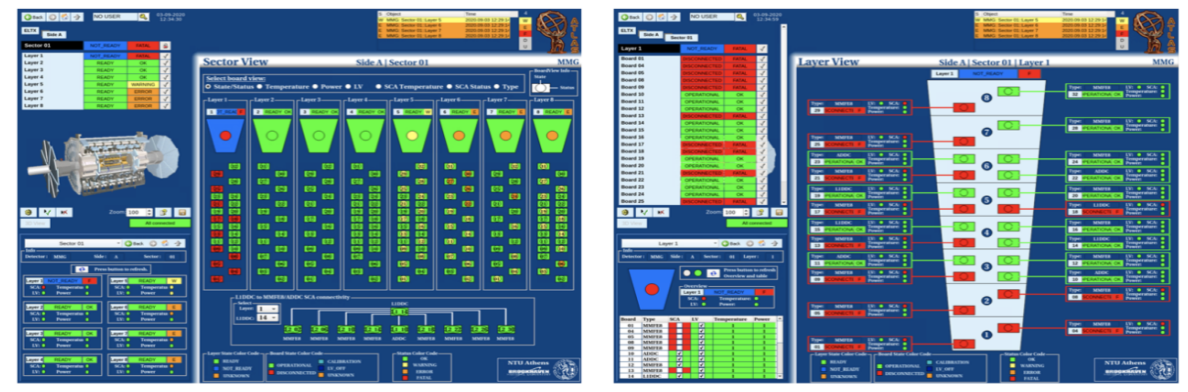

Figure 4: The DCS FSM and panels for the NSW Electronics monitoring

A common readout path and a separate trigger path are developed for each detector technology. The electronics design of such a system is implemented in about 8000 frontend boards including the design of a number of custom radiation tolerant ASICs [7] capable to drive trigger and tracking primitives to the backend trigger processor and readout system.

\subsubsection{Temperature and Magnetic field Monitoring}

NSW on-detector and cooling temperature monitoring relies on 64 MDT Device Modules (MDM) [8], 32 on each wheel. These devices will monitor the detector temperatures and magnetic field. MDMs are radiation and magnetic tolerant, based on Embedded Local Monitor Board technology and as such, will be attached on the wheels. Every NSW Sector will have 36 temperature sensors connected on the surface of each chamber and 16 in the cooling channels of each sector. Information about CanOpen OPC UA Server, which is the interface between hardware and projects, IP and $\mathrm{HO}$ wedges monitoring and cooling temperature sensors and individual alarm handling is included. The readout is implemented via the Controller Area Network (CAN) protocol. Each of the large sTGC sectors has four magnetic field sensors that are connected also on the MDM . 


\section{References}

[1] ATLAS Collaboration 2008, The ATLAS Experiment at the CERN Large Hadron Collider, JINST 3 S08003

[2] ATLAS Collaboration 2013, New Small Wheel Technical Design Report CERN-LHCC-2013O06 ATLAS-TDR-020, https://cds.cern.ch/record/1552862?ln=el

[3] T.Alexopoulos et al 2019, Performance studies of resistive-strip bulk micromegas detectors in view of the ATLAS New Small Wheel upgrade, Nucl. Instrum. Meth. A 937125

[4] Barriuso A P 2008 et al. The detector control system of the atlas experiment, JINST 3 P05006

[5] Holme O et al. 2005, The JCOP framework ICALEPCS Conf.Proc.C051010:WE2.1-6O, https://inspirehep.net/literature/699121

[6] CAEN SpA Power Supply systems, https://www.caen.it

[7] Caratell A et al. 2015, The GBT-SCA, a radiation tolerant ASIC for detector control and monitoring applications in HEP experiments, JINST 10 C03034

[8] Robert Hart, The ATLAS MDT Control System, Proceedings of ICALEPCS2009 TUP079, https://accelconf.web.cern.ch/icalepcs2009/papers/tup079.pdf 